\title{
Effective Dual Decomposition Methods in Network Traffic Management with Multipaths
}

\author{
S Thirunavukkarasu, K.P.Kaliyamurthie
}

\begin{abstract}
In today's Internet, Traffic management has met the unhealthy operation. To get the healthy operation of an internet, they have developed several traffic management tools. Such tools are together with congestion control at end hosts, routers get the routing protocol and network operators using traffic engineering. In this paper, we have used current innovations in optimization theory to perform a start to end reformat of traffic engineering. The objective function has been proposed based on goals of end users and network operators. Hence, we combined four distributed algorithms by using all known optimization decomposition methods, where source acclimate their sending rates through multipaths, according to different classes of feedback from the links. Finally, we applied TRUMP, recent traffic management protocol, which is acclimate and strong. With the help of above protocol, we can maximize the network utilities.
\end{abstract}

Keywords: Traffic engineering, network utility maximization, multipath routing, network resource management, TRUMP.

\section{INTRODUCTION}

Traffic management is the modification of origin levels and routing in order to be able to use network resources to handle customers and operators ' objectives. There are three performers in Internet Traffic management: users, routers, and operators. -client can conduct the congestion control at the edge of the network to change their sending rates[3]. An embedded device on the Web is the modem policy group, only a single network or a channel group, and switches operate the quickest path mapping premised on reply weights. In separate, network operators balance the traffic and minimize a cost function by tuning link weights, where the weight setting problem is truly NP-hard there is no practically optimal solution. [4][6]. Optimization decomposition, (e.g. $\mathrm{x}+\mathrm{y}+\mathrm{z}+\mathrm{w} \leq \mathrm{c}, \mathrm{x}+\mathrm{y} \leq \beta$ and $\mathrm{z}+\mathrm{w} \leq \mathrm{c}-\beta$, new variable $\beta$ updated by various methods) everyone can be solved locally. The different elements of traffic management were used effectively to analyze and design using scalability theory, which was also used to direct the design of new protocols for congestion control[3],[6],[9 ] and scalability theory was used to evaluate planned traffic engineering guidelines.

In our start to end redesign of traffic management, we should identify the objective function and constraints and derive distributed solutions using optimization decomposition practices. In which simulation has been used to comparing

Revised Manuscript Received on October 22, 2019.

S.Thirunavukkarasu, Department of Information Technology, Bharath Institute of Higher Education and Research, Tambaram, India.

Dr.K.P.Kaliyamurthie, Professor \& Dean, Department of Computer Science and Engineering, Bharath Institute of Higher Education and Research, Chennai, India performance properties of different solutions. Our contributions are two-fold:

Reconstructed Traffic Management: We introduce TRUMP: Determines traffic levels and splits assets, an easy-to-manage shared protocol that functions well for different topologies, capacities, delays in reviews and traffic volumes.

\section{RELATED WORKS}

\section{A. Choosing an objective function}

In this paper, we look for the best framework of the optimization problem. In the traffic management, routing and source rates are act as an optimization variable, resource allocation is more flexible. In our problem, the link load of constraint always should be lesser than the capacity, here topology and link capacities act as a constraint. The simulation provides the slow converge results and is perceptive to step size. From our observation, we introduce a different objective function by using dual decomposition problem [3][4]. The optimization practice allows us to derive a distributed solution to a given optimization problem, which will form the basis for multiple decompositions.

\section{B.Aggregate Utility Maximization}

The main balanced for the traffic management system is to maximize cumulative user utility, where each source s uses a utility function $\mathrm{U}_{\mathrm{s}}\left(\mathrm{x}_{\mathrm{s}}\right)$ as a function of its overall transmission rate $\mathrm{x}_{\mathrm{s}}$. Its utility functions $\mathrm{U}_{\mathrm{s}}$ are increasing, strictly concave, and double continuously differentiable function, e.g. $\log \left(\mathrm{x}_{\mathrm{s}}\right)$, that can also represent the resistance of the traffic or find out the fairness of resource allocation. This type of objective is covertly achieved by TCP congestion control today [6][12]. So, the optimization problem can be written as follows:

$$
\text { maximize } \sum \mathrm{U}_{\mathrm{s}}\left(\mathrm{x}_{\mathrm{s}}\right) \text { subject to } \mathrm{Rx} \leq \mathrm{c}, \mathrm{x} \geq 0
$$

Here both $\mathrm{R}$ and $\mathrm{x}$ represents variables. For single routing that is generally used in the current Internet, $\mathrm{R}$ is a $0-1$ matrix. Set $R_{l s}=1$ if link 1 is in a path of source $s$ and $R_{l s}=0$ otherwise. A single path routing only limits the achievable throughput. In the above problem (1) has a non-convex constraint set, which can be transformed into a convex set through the selection of multipath routing. To access multipath routing, we initiate $\mathrm{z}_{j}^{\mathrm{s}}$ to represent the sending rate of source $s$ on its jth path. We also represent available paths by a matrix $H$ where $H_{1 j}^{\mathrm{s}}=1$ if path $\mathrm{j}$ of source s uses link $\mathrm{k}$ and $\mathrm{H}_{\mathrm{lj}}^{\mathrm{s}}=0$ otherwise. $\mathrm{H}$ does not necessarily present all possible paths in the physical topology, but a subset of paths chosen by operator or the routing protocol [6]. 
Then we can rewrite the Multipath Utility Maximization as:

$$
\begin{gathered}
\operatorname{maximize} \sum_{s} U_{s}\left(\sum_{j} z_{j}^{s}\right) \\
\text { subject to } \sum_{s} \sum_{j} H_{l j}^{s} z_{j}^{s} \leq c_{l}, \forall l
\end{gathered}
$$

\section{Dual Decomposition Problem}

The above problem states that convex optimization problem structure is used for deriving a distributed solution to (2) by using dual decomposition. The primal variable $\mathrm{z}$ is appeared in the original problem.[3] To relax the constraint, dual variables can be introduced. The Lagrangian method can be applied to (2) as given below:

$$
\begin{gathered}
\mathrm{L}(\mathrm{z}, \mathrm{p})=\sum_{s} U_{s}\left(\sum_{j} z_{j}^{s}\right)+\sum_{l} p_{l}\left(c_{l}-y_{l}\right) \\
\mathrm{L}(\mathrm{z}, \mathrm{p})=\sum_{s} U_{s}\left(\sum_{j} z_{j}^{s}\right)+\sum_{l} p_{l}\left(c_{l}-\sum_{s} \sum_{j} H_{l j}^{s} z_{j}^{s}\right) \\
=\sum_{s}\left[U_{s}\left(\sum_{j} z_{j}^{s}\right)-\left(\sum_{l} \sum_{j} H_{l j}^{s} p_{l}\right) z_{j}^{s}\right]+\sum_{l} c_{l} p_{l}
\end{gathered}
$$

$$
\text { Let } \mathrm{Q}_{\mathrm{s}}=\sum_{l} \sum_{j} H_{l j}^{s} p_{l} \text { is the point-to point price for }
$$

source s.

The Lagrange dual function $\mathrm{g}(\mathrm{p})$ is specified as the highest $\mathrm{L}(\mathrm{z}, \mathrm{p})$ over $\mathrm{z}$ for given $\mathrm{p}$. Each source considering the cumulative link price $q_{s}$ is source $\mathrm{s}$ of feedback which does the aggregated utility maximization distributed process:

$$
z_{s}^{*}\left(q_{s}\right)=\underset{z_{j}^{s}}{\arg \max }\left[U_{s}\left(z_{j}^{s}\right)-Q_{s} z_{j}^{s}\right], \forall s
$$

The Lagrangian dual function $g(p)$ is defined as the maximized $\mathrm{L}(\mathrm{z}, \mathrm{p})$ over $\mathrm{z}$ for the given $\mathrm{p}$. Optimization performs the computation of source by $z^{s^{*}}(s)$. The Lagrange dual problem of (3) is:

minimize $\mathrm{h}(\mathrm{s})=L\left(z^{8}(s), s\right)$

subject to $s \geq 0$.

Through each relation it can be solved locally. It can be noticed that a convex minimization is above problem (4). If the problem (4) can not be distinguished, an iterative sub-gradient method can be used on each reference to update the dual variable $\mathrm{p}$ in parallel to solve the dual problem:

$$
p_{l}(t+1)=\left[p_{l}(t)-\beta_{p}(t)\left(c_{l}-\sum_{s} \sum_{j} H_{l j}^{s} z_{j}^{s}(t)\right)\right]^{+}
$$

where $\beta_{p}(t)$ represents dual variable step size, and $\mathrm{t}$ represents the iteration number and $\beta_{p}(t)>0$
At this point, $\mathrm{t}$ stands for iteration number which depends upon the longest Round-Trip Time of the network. So, every iteration receives the same timescale.

\section{E. Appended objective function for Traffic}

\section{Management}

To avoid the worst convergence properties of DUMP, another problem formulation is introduced to explain the operator's objective function.

minimize $\sum_{l} f\left(\sum_{s} R_{l s} x_{s} / c_{l}\right)(5)$ In current traffic engineering practices, the above optimization problem is solved in which $\mathrm{R}$ is variable and $\mathrm{x}$ is constant. Here $\mathrm{f}$ is denoted as a convex, non-decreasing, and twice differentiable function that gives progressively heaver penalty as link load increases, e.g., $e^{\sum_{s} R_{l s} x_{s} / c_{l}}$. The two-fold complexity chooses f. Firstly, this models M/M/1 Queuing delay [3][5]. Secondly network operators want to compliment solutions with many links which are closer to capacity. They do not bother whether a link is $20 \%$ loaded or $40 \%$ loaded. If it is solved (5) with both $\mathrm{x}$ and $\mathrm{R}$ as variables, then the solution will end up provided there is no traffic, which is also objectionable.

\section{MULTIPLE DECOMPOSITIONS}

In this section, we illustrate the distributed algorithms developed from all known optimization decompositions of (6). First the problem (5) is converted to a convex optimization problem using $\mathrm{z}$ and $\mathrm{H}$.

$$
\begin{gathered}
\operatorname{maximize} \sum_{s} U_{s}\left(\sum_{j} z_{j}^{s}\right)-w \sum_{l} f\left(y_{l} / c_{l}\right) \\
\text { subject to } \mathrm{y} \leq \mathrm{c}, \quad(6) \\
y_{l}=\sum_{s} \sum_{j} H_{l j}^{s} z_{j}^{s}, \forall l .
\end{gathered}
$$

In the above problem, $\mathrm{U}$ and $\mathrm{f}$ represents source and link functions. An added variable $\mathrm{y}_{l}$ is introduced to offer feedback earlier link load exceeds the genuine capacity. Based on this algorithm, the path rate resulting solutions are updated according to the feedback prices from links [4].

\section{A.Effective Capacity}

The researcher has used three major algorithms partial dual, primal dual and full dual for avoiding link loads from link capacity by supplying feedback based on effective capacity instead of actual capacity [3][6]. In the resulting algorithm, the sources update their path rates based on feedback price just as in Fig 1. The feedback price is equivalent to Fig 1 . Except effective capacity $y_{l}$. A single dual variable $\mathrm{p}$ is introduced to relax the constraint $y_{l}=\sum_{s, j} H_{l j}^{s} z_{j}^{s}$

The feedback price is updated as follows:

$$
p_{l}\left(t+T_{p}\right)=p_{l}(t)-\beta_{p}\left(y_{l}(t)-\sum_{s} \sum_{j} H_{l j}^{s} z_{j}^{s}(t)\right.
$$


In seeing constant step sizes in this section, the $t$ argument is removed from all the step sizes. Equation (8) is identical to Fig 4.2, except using the effective capacity instead of actual capacity. This is one way to provide feedback to the source before the links reach the actual capacity.

\section{B.Local Optimization: Partial-dual}

The Partial-dual algorithm derives a new one which is except with effective capacity $y$ as an additional primal variable. The constraint $\mathrm{y} \leq \mathrm{c}$ is required to get the resulting in the following equation for updating effective capacity:

$$
y_{l}\left(t+T_{p}\right)=\underset{\left(y_{l} \leq c_{l}\right)}{\min \operatorname{imize}_{\mathrm{j}}}\left(y_{l} / c_{l}\right)-p_{l}(t) y_{l}
$$

\section{C.Subgradient Restore: Primal-dual} problems by using the primal dual algorithm where one is responsible for each primal variable. The master problem solves for $\mathrm{y}$ assuming a given $\mathrm{x}{ }^{*}$ while the sub problem solves for $\mathrm{x}$ assuming a fixed $\mathrm{y}$.

\section{D.Consistency Price: Full Dual}

The full dual introduced second dual variable $\mathrm{q}$ to relax the constraint $\mathrm{y} \leq \mathrm{c}$. This dual variable can be interpreted as consistency price as it ensures consistency between the effective capacity and the capacity constraint at the stability point. With the feedback price, the consistency price is updated over time using a Subgradient method:

$$
q_{l}\left(t+T_{p}\right)=\left[q_{l}(t)-\beta_{p}\left(c_{l}-y_{l}(t)\right)\right]^{+}
$$

Where $\beta_{p}$ is the step size for consistency price.The capacity constraint is braked the consistency price only to arrive hooked on play. Hence, it will plot a non-negative value. The effective capacity update is based on both link prices.

$$
y_{l}\left(t+T_{p}\right)=\underset{y_{l}}{\min \text { imize }_{\mathrm{z}}} \mathrm{wf}\left(y_{l} / c_{l}\right)-\left(p_{l}(t)+q_{l}(t)\right) y_{l}
$$

\section{E. Path Rate update in Direct: Primal Driven}

All the previous algorithms were introduced axillary dual variables to relax the constraints. In this primal decomposition algorithm, a direct solution can easily be found by introducing a penalty function. Here applied penalty function $k_{l}\left(\sum_{s} \sum_{j} H_{l j}^{s} z_{j}^{s}\right)$ replace the capacity constraint $\mathrm{Hz} \leq \mathrm{c}$. Where the penalty function is a continuous, increasing, differentiable and convex function that is enough. Link loads will not exceed the link capacity. The optimal point will not affect if the link loads are enough and close to zero for values less than capacity

\section{RESULTS AND DISCUSSION}

In this section, first, we determine the speed of convergence and the feasible maximum utility then adjust the best level. Second, we determine algorithms to get the variable updated faster convergence. It also uses the local minimization instead of constant updated faster convergence.
First decomposes the single problem (7) into two sub

Third, we determine the consistency price which can give convergence for small parameter $\mathrm{w}$.

\section{A. Combination of MATLAB Experiments}

The number of tuning parameters has been used to find the optimal value. MATLAB simulations are used with the help of various topologies. Then, the traffic patterns are used to analyse the structure of properties that will perform the better convergence. For each algorithm, the changes of topology source and link variables can be made at each iteration. It depends upon the link load with the comparison of existing iteration. For the utility function U, logarithmic function is used for getting the utility maximization result. For the cost function $\mathrm{f}$, the researcher has used an exponential function to receive the minimum cost.

We apply one best real time topologie as shown in Figure 1. Here Figure 1 is an Access Core topology. For the given topology, we choose six source-destination pairs (1-8, 2-5, 4-10, 4-12, 5-2, 5-3) for Access Core topology The simulations consider the link capacities should follow the positive values only when we are using the Gaussian distribution. It needs to omit the negative values for getting the optimal result. Among these experiments, we determine $99.8 \%$ convergence result of the optimal aggregate utility of (6). The initial independent rates are assigned to getting the convergence rates.

\section{B. Evaluating User Utility and Operator Cost}

In this section, we examine the resolution between maximum utility and time of convergence. In Figure1, we have used three different parameter values of $w$ for the partial dual algorithm to plot the number of iterations before convergence across step size $\beta_{s}$. Each point be consistent to several capacity values for each stepsize and the average number of iterations to be used previously convergence is displayed in a solid line.

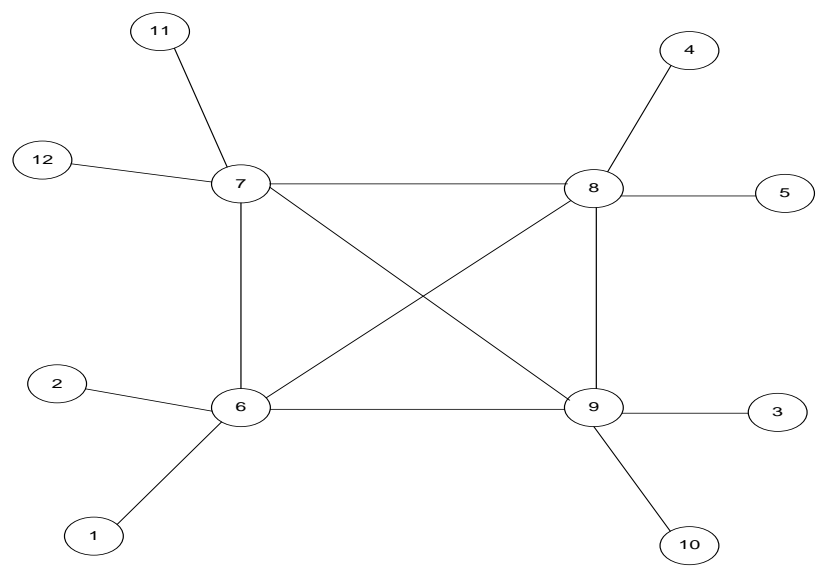

Figure 1: Access-Core Topology 


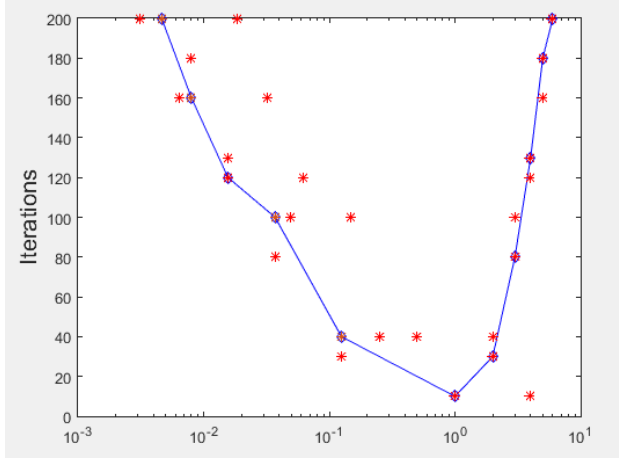

Figure 1: Plots of partial-dual algorithm

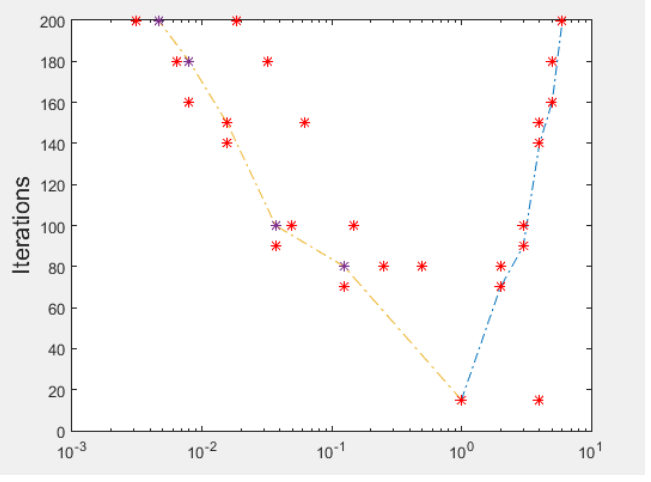

Figure 2: Plots of partial-dual algorithm

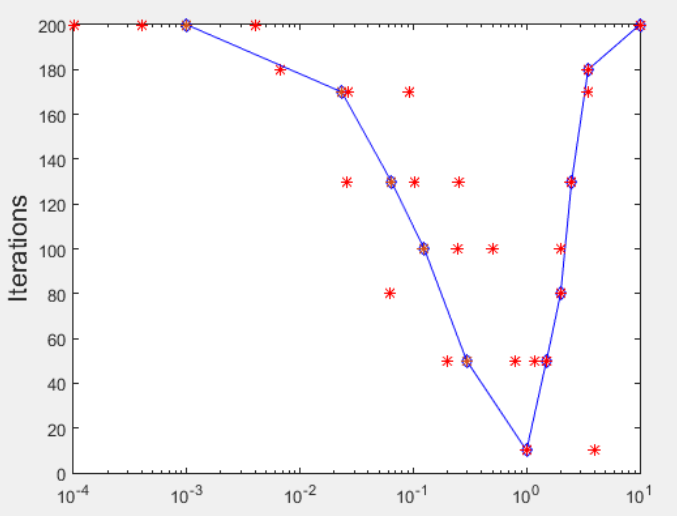

Figure 3: Plots of partial-dual algorithm appearing based on convergence time on stepsize and parameter value $\mathbf{w = 1 / 3 6}$ for Access-core topology.

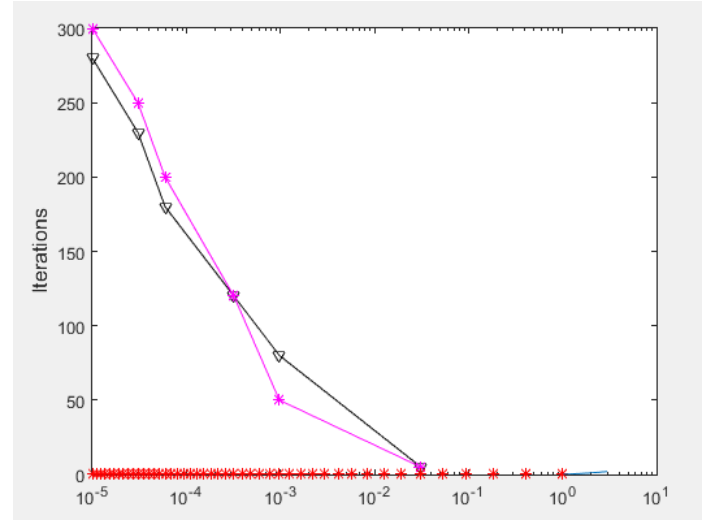

Figure 4: Plots of TRUMP algorithm appearing based on convergence time on stepsize and using parameter values $w=1,1 / 6$ and 1/36 for Access-core topology.

In the above graph, we used TRUMP algorithm to get the better result of convergence time according to the stepsize by using the various parameter values $\mathrm{w}=1,1 / 6,1 / 36$ for Access-core topology. The parameter values are applied in equation (1) to plot a graph. This equation given the optimal results for given topology and a range of parameter values.

Table 1: Summary of Aggregate form of Iterations to convergence for perfect selected Tuning Parameters. Here * represents the Sensitivity for stepsize deviation and ** represents added sensitivity for stepsize deviation.

\begin{tabular}{|c|c|c|c|c|c|}
\hline Algorithms & Parameters & Primal-Dual & Partial-Dual & Full-Dual & Primal \\
\hline Access-Core & $1 / 3$ & $125^{*}$ & $80^{* *}$ & $110^{*}$ & $130^{*}$ \\
\hline Access-Core & $1 / 6$ & $125^{*}$ & $85^{* *}$ & $150^{*}$ & $110^{*}$ \\
\hline Access-Core & 1 & $25^{*}$ & 20 & 15 & 25 \\
\hline
\end{tabular}

\section{CONCLUSION}

This paper examined different network utility maximization and congestion control algorithms such as partial dual, primal dual, primal driven and full dual for avoiding link loads. we have explored the ideas of existing and new systems to improve network utility maximization such as optimal throughput, fairness, link utilization, convergence speed. It is necessary to include the traffic management protocol that is distributed, adjust, powerful, flexible and easy to control. The partial dual algorithm obtained maximal aggregate utility results when the parameter values are smaller than the knee region.

\section{REFERENCES}

1. BAPATLA, D. and PRAKRIYA, S., 2019. Performance of a Cooperative Network with an Energy Buffer-Aided Relay. IEEE Transactions on Green Communications and Networking, 3(3), pp. 774-788.

2. USHA, M. and RAMAKRISHNAN, B., 2019. A Robust Architecture of the OLSR Protocol for Channel Utilization and Optimized Transmission Using Minimal Multi Point Relay Selection in VANET. Wireless Personal Communications, 109(1), pp. 271-295.

3. DEVI, B.R., RAO, K.K. and RANI, M.A., 2019. Application of Modified Bellman-Ford Algorithm for Cooperative Communication. Wireless Personal Communications, 109(3), pp. 2025-2049.

4. NGUYENA, T.N., TRANC, M., DUY-HUNG, H., TRANGE, T.T and VOZNAK, M., 2019. Multi-source in DF cooperative networks with the PSR protocol based full-duplex energy harvesting over a Rayleigh fading channel: Performance analysis. Proceedings of the Estonian Academy of Sciences, 68(3), pp. 264-275.

5. TANG, K. and LIAO, S., 2020. Buffer-aided cooperative spectrum sharing with full-duplex wireless-powered relay. Computer Networks, 166

6. TYAGI, A., SINGH, A. and BHULANIA, P., 2019. Partner Selection Based on Different Protocols in Cooperative Network by Using Nakagami-M Function, 2019 6th International Conference on Signal Processing and Integrated Networks, SPIN 2019 2019, pp. 568-573. 
7. FU, M. and WU, F., 2017. Investigation of multipath routing algorithms in software defined networking, Proceedings - 2017 International Conference on Green Informatics, ICGI 2017 2017, pp. 269-273.

8. HAMZEH, H., HEMMATI, M. and SHIRMOHAMMADI, S., 2017. Priced-Based Fair Bandwidth Allocation for Networked Multimedia, Proceedings - 2017 IEEE International Symposium on Multimedia, ISM 2017 2017, pp. 19-24.

9. KHOSRAVI, M.R., BASRI, H. and ROSTAMI, H., 2018. Efficient routing for dense UWSNs with high-speed mobile nodes using spherical divisions. Journal of Supercomputing, 74(2), pp. 696-716.

10. LIN, Y., LIU, T., WANG, S. and LAI, Y., 2019. Proactive multipath routing with a predictive mechanism in software-defined networks. International Journal of Communication Systems, 32(14),

11. TANESSAKULWATTANA, S. and PORNAVALAI, C., 2019 Multipath energy balancing for clustered wireless sensor networks. Wireless Networks, 25(5), pp. 2537-2558.

12. ZAW, H.T. and MAW, A.H., 2019. Traffic management with elephant flow detection in software defined networks (SDN) International Journal of Electrical and Computer Engineering, 9(4), pp. 3203-3211.

13. FU, X., FORTINO, G., PACE, P., ALOI, G. and LI, W., 2020 Environment-fusion multipath routing protocol for wireless sensor networks. Information Fusion, 53, pp. 4-19.

\section{AUTHORS PROFILE}

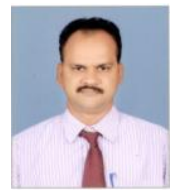

S. Thirunavukkarasu, Assitant Professor, Department of Information Technology, Bharath Institute of Higher Education and Research, Chennai, India

Dr.K.P.Kaliyamurthie is self- directed, enthusiastic educator with a commitment on student development. $\mathrm{He}$ is with Bharath University, Chennai, Tamil Nadu, India as Professor and Dean of Computer Science and Engineering. He has over 29 years of rich experience in teaching along with student administration. 